\title{
A link between students' discernment of variation in unidirectional change and their use of quantitative variational reasoning
}

\author{
Heather Lynn Johnson ${ }^{1}$ • Evan McClintock ${ }^{1}$
}

Published online: 4 January 2018

(C) The Author(s) 2018. This article is an open access publication

\begin{abstract}
In this design experiment study, we investigated the problem: What conditions might foster students' discernment of a critical aspect — variation in unidirectional change (e.g., discerning a "decreasing" increase)? At a public middle school in a large US city, we led a sequence of three days of whole class lessons, followed by task-based, clinical interviews with 14 seventh grade students ( $\sim 13$ years old). Students interacted with researcher-developed dynamic computer environments, which linked filling polygon animations with dynamic graphs. We report results to demonstrate a range of students' work along with detailed analyses of student interview episodes. We found connections between students' predictions of graph type (linear, "mostly" linear, nonlinear), students' conceptions of attributes represented by a graph, and students' discernment of variation in unidirectional change. Students who discerned variation in unidirectional change also engaged in quantitative variational reasoning. To foster students' discernment of variation in unidirectional change, task designers should provide opportunities for students to conceive of attributes as capable of varying and possible to measure.
\end{abstract}

Keywords Mathematical reasoning · Design experiment - Variational reasoning · Technology · Secondary students

\section{Introduction}

Discerning variation in unidirectional change (e.g., discerning an "increasing" or "decreasing" increase) is challenging even for successful university students (Carlson, Jacobs, Coe, Larsen, \& Hsu, 2002), yet important for secondary students' study of key mathematical concepts such

Heather Lynn Johnson

Heather.johnson@ucdenver.edu

1 University of Colorado Denver, 1380 Lawrence Street, Room 731 Campus Box 106, P.O. Box 173364, Denver, CO 80217-3364, USA 
as function and rate (e.g., Ellis, Özgür, Kulow, Williams, \& Amidon, 2015; Herbert \& Pierce, 2012; Johnson, 2012; Lobato, Hohensee, Rhodehamel, \& Diamond, 2012). Researchers have identified factors that could mitigate students' discernment, including students' images of change and variation (Castillo-Garsow, Johnson, \& Moore, 2013; Thompson \& Carlson, 2017) and students' interpretation of graphs (Bell \& Janvier, 1981; Clement, 1989; Moore \& Thompson, 2015; Slavit, 1997). Drawing on Thompson's theory of quantitative reasoning (1994), we identify a factor affording students' discernment: students' conceptions of attributes as "things" that are capable of varying and possible to measure. We provide evidence of a link between students' conceptions of the possibility of measuring an attribute, students' conceptions of variation, and students' discernment of variation in unidirectional change.

Consider situations involving filling polygons, such that interior of a polygon fills as a segment parallel to a base moves along the polygon. Figure 1 shows two situations - a filling triangle (right) and a filling rectangle (left). For both the triangle and rectangle, a student might state: "as the shape fills, the area of the filled part increases." For the triangle, another student might state: "the amounts of increase in area become smaller as the triangle gets more filled." Both statements provide evidence of students' discernment of variation in an attribute (area) they conceive of as being possible to measure. The second statement provides evidence of a student's discernment of variation in unidirectional change.

Kaput (1994) argued that dynamic computer environments (DCEs), linking dynamic animations and graphs, could serve as rich settings for students to investigate the mathematics of change and variation. Researchers investigating secondary students' conceptions of function, rate, and variation have made extensive use of DCEs in their studies (e.g., Ellis et al., 2015; Herbert \& Pierce, 2012; Johnson, 2012; Lobato et al., 2012; Saldanha \& Thompson, 1998). When interacting with DCEs representing varying rates of change, secondary students have shown evidence of discerning variation in unidirectional change (Ellis et al., 2015; Johnson, 2012; Saldanha \& Thompson, 1998). Using Marton's variation theory (2015), we frame our design of filling polygon DCEs and related tasks, which we implemented to provide students opportunities to discern variation in unidirectional change. Using Thompson's theory of quantitative reasoning (1994), we incorporated attributes - length, height, and area - which we anticipated students could conceive of as capable of varying and possible to measure.

Area $E F G H=4.12 \mathrm{~cm}^{2}$

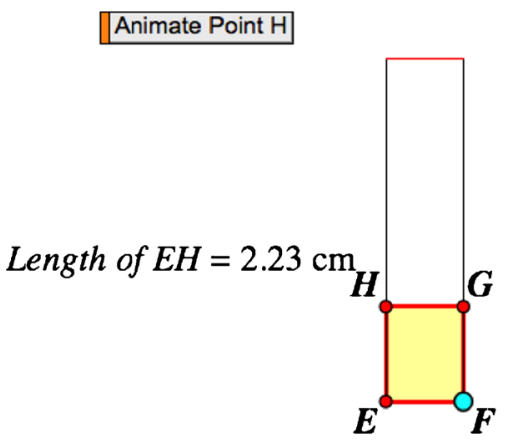

Length of $E F=1.85 \mathrm{~cm}$
Area $A B C D=8.97 \mathrm{~cm}^{2}$

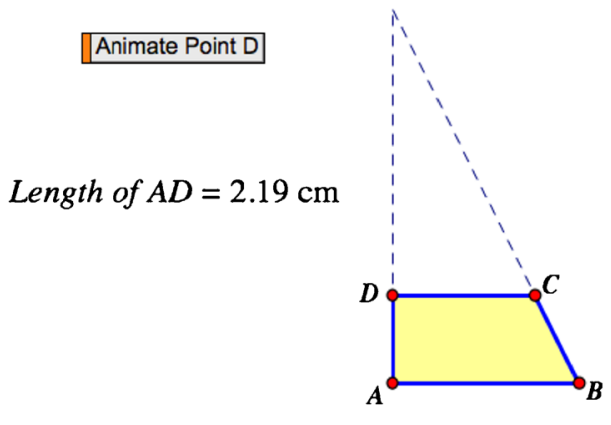

Length of $A B=4.65 \mathrm{~cm}$

Fig. 1 Filling rectangle (left) and filling triangle (right) 
Researchers have shown the utility of variational reasoning for fostering early secondary students' conceptions of function and interpretations of graphs (Ayalon, Watson, \& Lerman, 2015; Ellis et al., 2015; Hitt \& González-Martín, 2015; Johnson, 2012; Lobato et al., 2012). We use Thompson's theory of quantitative reasoning (1994) to frame our perspective on variational reasoning. Students' variational reasoning consists of two components: students' images of change and students' conceptions of attributes (Castillo-Garsow et al., 2013; Thompson \& Carlson, 2017). We use the term quantitative variational reasoning (QVR) to mean students' reasoning about attributes that they can conceive of as capable of varying and possible to measure.

In this design experiment (Cobb, Confrey, diSessa, Lehrer, \& Schauble, 2003) study, we address the problem: What conditions might foster students' discernment of variation in unidirectional change? We use grounded theory (Corbin \& Strauss, 2008) analysis methods to link students' use of QVR with their discernment of variation in unidirectional change. We report results to demonstrate the range of students' reasoning, along with student interview episodes with a researcher-developed DCE and related tasks. We address implications for task design to foster students' discernment of variation in unidirectional change.

\section{Background}

\subsection{Conceptual and theoretical framework}

We employ a theoretical framework that networks theories of different grain sizes (Johnson, McClintock, Hornbein, Gardner, \& Grieser, 2017). Broadly, this theoretical framework informed our design within and across tasks intended to foster students' discernment of variation in unidirectional change (Fig. 2). By tasks we mean both problem situations and students' conceptions of those problem situations. We used Marton's variation theory (2015) to frame our design across tasks. We used Thompson's theory of quantitative reasoning (1994) to frame our perspective of student conceptions of task elements. With the vertical arrows in Fig. 2, we show how Thompson's theory of quantitative reasoning informed our perspective of what constitutes a critical aspect. In this section, we articulate key components of QVR; then, we elaborate on the critical aspect (Marton, 2015) that we intended for students to discern: variation in unidirectional change. In Section 3.5 we explain how we used variation theory to frame our design across tasks.

QVR has two key components: students' conceptions of attributes as being capable of varying (variational component) and possible to measure (quantitative component). The variational component of QVR entails students' images of change, which extend beyond static mental pictures to encompass students' dynamic mental activity (Thompson, 1996). Two such images of change are chunky and smooth (Castillo-Garsow et al., 2013). For example, a chunky image of change involves conceiving of completed amounts of change (e.g., area changed by $a$ units and height changed by $b$ units). In contrast, a smooth image of change involves conceiving of continuing change (e.g., area changing in conjunction with changing height). Consequently, students' variational reasoning can take different forms depending on students' images of change (Thompson \& Carlson, 2017).

The quantitative component of QVR entails students' conceptualization of an attribute as being possible to measure. Following Thompson (1994), we use the term quantity to mean an 


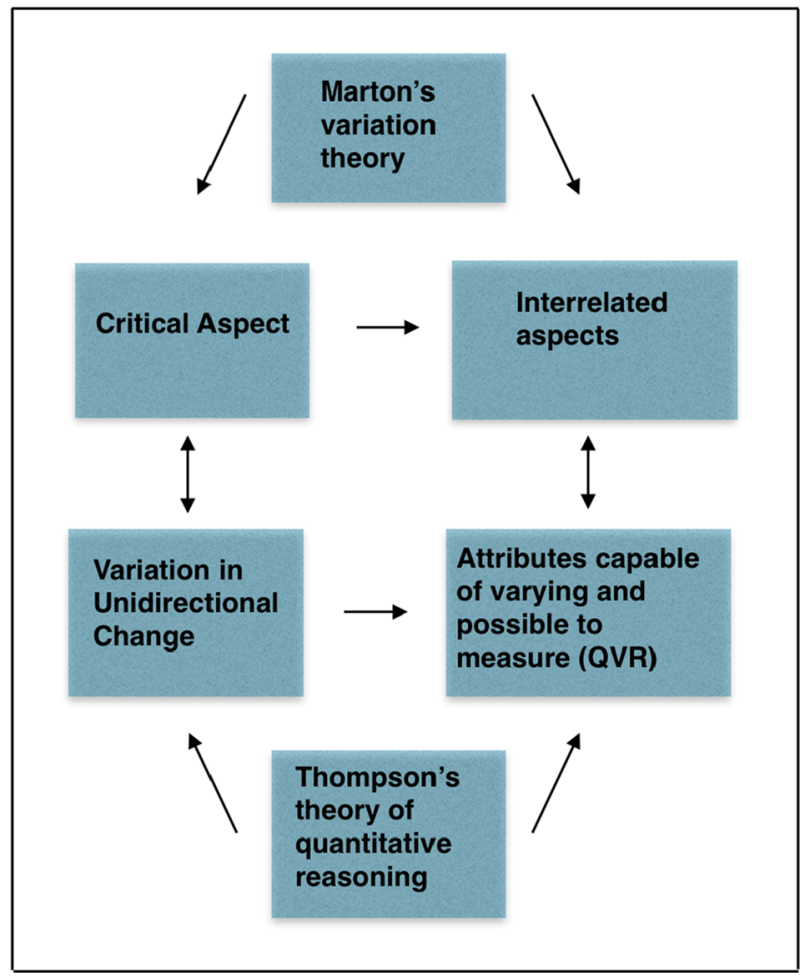

Fig. 2 Networking theories to design across and within tasks

individual's conception of the possibility of measuring some attribute of an object. Conceiving of the possibility of measuring an attribute is distinct from noticing a physical attribute of an object. To illustrate, a student might conceive of the pointiness of a triangle, as an attribute possible to measure. Furthermore, the student may conceive of the possibility of measurement without actually engaging in measuring a triangle's pointiness. In contrast, a student conceiving of pointiness as a physical attribute of a triangle might notice differences in the pointiness of two triangles. Yet, that student may not be conceiving of pointiness as something that is possible to measure, just as a noticeable aspect of a triangle. Through these examples, we intend to illustrate that measurability is not inherent in an attribute; rather, it depends on how an individual conceives of the attribute.

Discernment of variation in unidirectional change entails conceiving of the possibility of varying the intensity of the change, while holding the direction of the change invariant. By discernment, we mean a student's attention to a situation in terms of certain critical aspects (Marton, 2015). By intensity, we mean the "degree to which an attribute is present" (Johnson, 2015a, p. 66). For the filling triangle (Fig. 1, right), students may provide evidence of discernment of different degrees of unidirectional change through statements such as: "the area starts to increase more slowly as the shape becomes more filled." To clarify, we distinguish students' conceptions of variation (students' dynamic mental activity entailing their images of change) from the variation central to Marton's variation theory. The object we intended for students to discern entails students' conceptions of variation - specifically, variation in unidirectional change. 


\subsection{Variational reasoning and images of change}

Drawing on Thompson and Carlson (2017), we distinguish variational reasoning from covariational reasoning. Variational reasoning entails conceiving of variation in values of some attribute of an object. Covariational reasoning entails the coordination of variation in values of two different attributes. Furthermore, we use the variation framework posited by Thompson and Carlson (2017) to distinguish levels of students' variational reasoning: at the highest level, smooth continuous variation, and at a lower level, gross variation. A student employing gross variation could conceive of variation in some attribute (e.g., area is increasing). In contrast, a student employing smooth continuous variation could use smooth images of change to conceive of variation in values of some attribute (e.g., conceiving of amounts of area as increasing). A student discerning variation in unidirectional change could engage in variational reasoning at either of these levels.

Researchers have found that early secondary students can discern variation in unidirectional change when employing chunky images of change (Ellis et al., 2015; Lobato et al., 2012). Given a situation involving a plant's height growing exponentially with time, Ellis et al. (2015) found that early secondary students conceived of variation in fixed amounts of increases in height and time, thereby demonstrating chunky images of change. Although it may be challenging for early secondary students to draw on smooth images of change (e.g., Ellis et al., 2015), such opportunities are important and needed, in part because of the role students' images of change can play in their study of function and interpretation of graphs (see CastilloGarsow et al., 2013; Johnson, 2012, 2015b; Saldanha \& Thompson, 1998).

\subsection{Covariation, functions, and graphs}

Researchers have shown that secondary students benefit from opportunities to use a covariation perspective when studying function (e.g., Ayalon et al., 2015; Bell \& Janvier, 1981; Confrey \& Smith, 1995; Hitt \& González-Martín, 2015; Saldanha \& Thompson, 1998). Saldanha and Thompson (1998) argued that key to a covariation perspective is a student's conception of covariation between quantities. Following Saldanha and Thompson (1998), a student using a covariation perspective could conceive of functions as specialized relationships between quantities. To provide students opportunities to use a covariation perspective on function, researchers have incorporated tasks involving dynamic and static Cartesian graphs (e.g., Ellis et al., 2015; Hitt \& González-Martín, 2015). Yet, students may interpret graphs in terms of something other than covariation, which may constrain the utility of certain tasks involving Cartesian graphs.

Researchers have explicated impoverished ways in which students can interpret graphs (e.g., Clement, 1989; Leinhardt, Zaslavsky, \& Stein, 1990). For example, students may interpret a graph iconically, such that the shape of a graph would resemble an object. Moore and Thompson (2015) introduced the constructs of static and emergent shape thinking to explain conceptual operations underlying students' interpretation of graphs. A student engaging in static shape thinking would interpret a graph as a being a manipulable shape (e.g., a graph of an absolute value function as being a "V"), but not necessarily iconically (e.g., a graph representing change in a liquid's volume and height in a filling bottle as being shaped like a bottle (see also Johnson, 2015b)). In contrast, a student engaging in emergent shape thinking would interpret a graph as a trace representing a relationship between covarying quantities. When interpreting graphs, it is desirable for students to use a covariation perspective involving 
smooth images of change (e.g., Castillo-Garsow et al., 2013). Therefore, it is desirable to promote students' use of emergent, rather than static shape thinking when interpreting graphs (Moore \& Thompson, 2015).

\section{Method}

\subsection{Research questions}

We investigated two research questions:

RQ1: How do aspects of students' variational reasoning impact their discernment of variation in unidirectional change?

RQ2: How do students engage in variational reasoning when interacting with tasks involving DCEs including filling polygon animations and linked dynamic graphs?

\subsection{Design experiment methodology}

A purpose of design experiment research (Cobb et al., 2003) is to develop theory closely tied to practice. In this research, we theorize about a role of QVR in students' discernment of variation in unidirectional change. A key feature of design experiments is an iterative design process, in which researchers test evolving conjectures (Cobb et al., 2003). We tested conjectures during this study as well as conjectures based on results of Johnson's previous research $(2012,2015 \mathrm{a}, \mathrm{b})$. We conjectured that some attributes, such as volume, might be challenging for students to conceive of as possible to measure. Hence, we incorporated filling polygon DCEs and related tasks. Furthermore, we conjectured that interacting with DCEs involving different types of filling polygons (e.g., triangles vs. rectangles) might impact students' opportunities to discern variation in unidirectional change. Our conjectures influenced the design of the DCEs and related tasks for this study (see Sections 3.4 and 3.5).

\subsection{Setting, data collection, and participants}

We conducted this study at a public middle school to which students applied to attend. The school, located in a gentrifying part of a large US city, served primarily students who identified as Mexican-American and were not necessarily from the neighborhood directly surrounding the school. At the time of the study, $92 \%$ of students attending the school were eligible for free and reduced lunch and $94 \%$ of students identified as students of color.

We organized data collection in two phases. In the first phase, Johnson taught a sequence of three consecutive whole class lessons to four sections of seventh grade students $(\sim 13$ years old) enrolled in a pre-algebra course. In the second phase, which occurred the week following the lessons, 14 students participated in a clinical interview (Clement, 2000). Johnson conducted a total of seven interviews (one interview per pair). We selected students who participated actively during the whole class lessons, including at least one pair of students from each section. McClintock served a supporting role during the lessons and interviews.

We designed this study to complement students' regular classroom instruction. Hence, we worked to balance a classroom teacher's pedagogical goals along with our research aims. 
Together with the students' regular classroom teacher, we agreed that whole class lessons taught by Johnson would focus on linear situations, which students had studied earlier in the year. Therefore, we used only the filling rectangle DCE (Section 3.4) during the whole class lessons. In the clinical interview, we used both the filling rectangle and filling triangle DCEs (Section 3.4).

Prior to this study, the students with whom we worked had not yet had opportunities to interact with DCEs and related tasks like the ones we incorporated. In addition, students had not yet explored nonlinear situations. Our purpose in the interview was to extend beyond students' work during the whole class lessons. We began each interview with tasks involving the filling rectangle DCE then moved to tasks involving the filling triangle DCE. The reported task sequence (Section 3.5) reflects our adjustments and refinements that occurred through our iterative design process.

\subsection{Dynamic computer environments}

Taking inspiration from Thompson, Byerly, and Hatfield's (2013) dynamic version of the wellknown bottle problem (Shell Centre for Mathematical Education (University of Nottingham), 1985), Johnson used Geometer's Sketchpad Software (Jackiw, 2009) to develop DCEs involving filling polygon animations. Through filling polygon DCEs, we intended to maintain the spirit of the bottle problem, while incorporating attributes of area and height, rather than volume and height. The DCEs consisted of linked animations and graphs representing the area and height of a filling rectangle and triangle (Figs. 3 and 4).

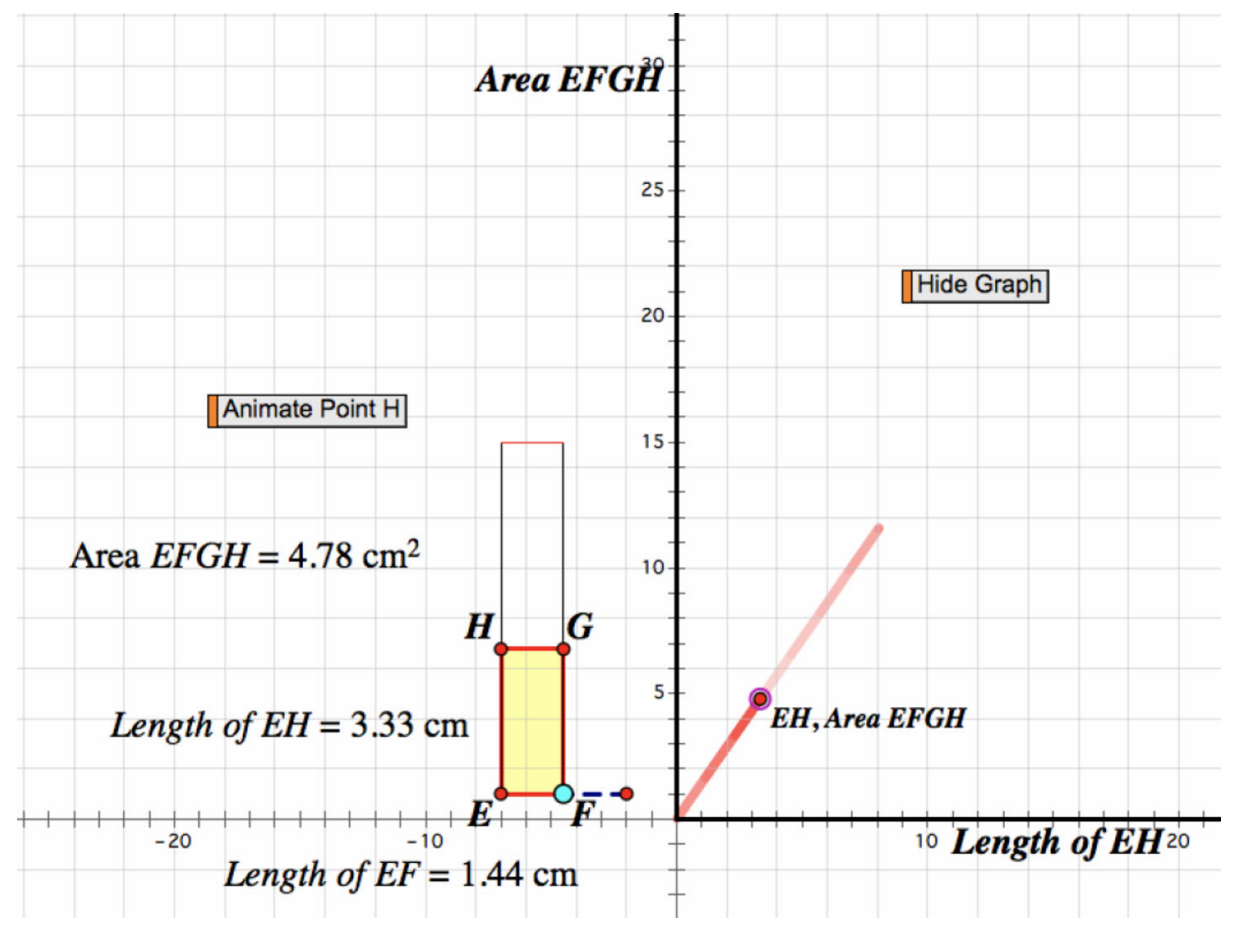

Fig. 3 Filling rectangle 


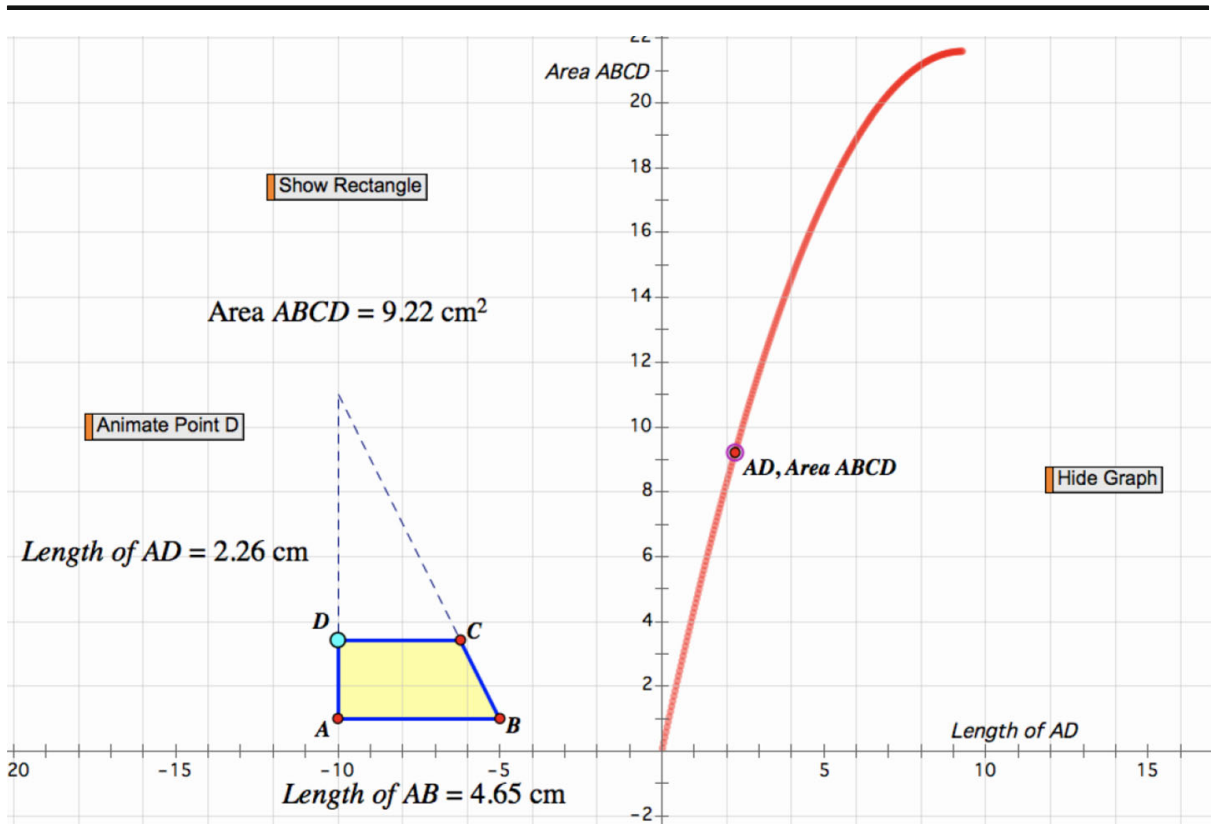

Fig. 4 Filling triangle

For the filling rectangle (Fig. 3), students could click and drag point $\mathrm{F}$ to vary the length of the base (EF). For the filling triangle (Fig. 4), the length of the base (AB) was fixed. To "fill" the rectangle or triangle, students could click and drag on points $\mathrm{H}$ or $\mathrm{D}$, respectively, or press the action button Animate Point $H$ (or D). If a student pressed animate point when EH (or AD) was greater than zero, the polygon would fill until point $\mathrm{H}$ (or D) reached the maximum height of the rectangle (or triangle), then reset $\mathrm{EH}$ (or $\mathrm{AD}$ ) to zero and begin filling again until the original length of $\mathrm{EH}$ (or AD) was reached. As the polygon fills, the linked graph dynamically traces.

\subsection{Task design and sequencing}

Table 1 shows the task sequence during the whole class lessons and subsequent interview. The middle column of Table 1 describes the specific task components. The left and right columns show the DCEs (filling rectangle, filling triangle) and attributes (height, base) that we varied.

The critical aspect we intended for students to discern-variation in unidirectional change - is comprised of interrelated aspects. In our set of tasks, we aligned with Marton's (2015) recommendations for task designers intending to foster students' discernment of critical aspects comprised of interrelated aspects: (1) Vary individual aspects, then both aspects, against a background of invariance; (2) Repeat patterns of variance and invariance with different backgrounds. In the filling rectangle tasks, we varied the base of the rectangle (whole class day 1), then the height of the rectangle (whole class day 2), and then both the base and height (whole class day 3/interview part 1). In interview part 2, we provided students with a 
Table 1 Task sequence during whole class lessons and subsequent interview

\begin{tabular}{|c|c|c|c|c|c|c|c|}
\hline Setting & \multicolumn{6}{|c|}{ Description of tasks } & $\begin{array}{c}\text { Feature } \\
\text { we varied }\end{array}$ \\
\hline \multirow{3}{*}{$\begin{array}{l}\text { Whole class } \\
\text { day } 1 \\
\text { Filling }\end{array}$} & \multicolumn{6}{|c|}{$\begin{array}{l}\text { Given non-well-ordered tables for different bases (EF), determine different amounts } \\
\text { of area. For example: The length of } E F \text { is } \mathbf{c m} \text {. Complete the table: }\end{array}$} & \multirow{4}{*}{$\begin{array}{l}\text { Length of } \\
\text { EF (base) }\end{array}$} \\
\hline & Length of EH & $1 \mathrm{~cm}$ & $3 \mathrm{~cm}$ & $5 \mathrm{~cm}$ & $7 \mathrm{~cm}$ & $10 \mathrm{~cm}$ & \\
\hline & Area of rectangle EFGH & & & & & & \\
\hline \multirow{4}{*}{$\begin{array}{l}\text { Whole class } \\
\text { day } 2 \\
\text { Filling } \\
\text { rectangle }\end{array}$} & \multicolumn{6}{|c|}{$\begin{array}{l}\text { Suppose we were to create a graph relating the side length EH and the area of EFGH. } \\
\text { Would the graph be linear? Explain why or why not. }\end{array}$} & \\
\hline & \multirow{3}{*}{\multicolumn{6}{|c|}{$\begin{array}{l}\text { Given the two different lengths of } \mathrm{EH} \text {, determine the amount of increase in area of } \\
\text { rectangle EFGH for lengths of } \mathrm{EH} \text { not given in the non-well ordered tables. } \\
\text { For example, The length of EF is } 3 \mathrm{~cm} \text {. Complete the table: }\end{array}$}} & \multirow{3}{*}{$\begin{array}{l}\text { Length of } \\
\text { EH } \\
\text { (height) }\end{array}$} \\
\hline & & & & & & & \\
\hline & & & & & & & \\
\hline $\begin{array}{l}\text { Whole class } \\
\text { day } 3 \\
\text { Filling } \\
\text { rectangle }\end{array}$ & \multicolumn{6}{|c|}{$\begin{array}{l}\text { Given a graph relating the side length } \mathrm{EH} \text { and the area of } \mathrm{EFGH} \text {, Determine at least } \\
\text { two different ways to complete the statement: When the length of side EH } \\
\text { increases by the area increases by } \\
\text { Suppose we were to create different graphs for rectangles with different lengths of EF. } \\
\text { What would those graphs be like? }\end{array}$} & $\begin{array}{l}\text { Length of } \\
\text { EH } \\
\text { (height) } \\
\text { Length of } \\
\text { EF (base) }\end{array}$ \\
\hline $\begin{array}{l}\text { Interview } \\
\text { part } 1 \\
\text { Filling } \\
\text { rectangle }\end{array}$ & \multicolumn{6}{|c|}{$\begin{array}{l}\text { Given graphs representing the area of EFGH as a function of the length of side EH, } \\
\text { determine the length of the base of the rectangle (EF). } \\
\text { What would a graph be like for a rectangle with a (1) short base, (2) long base? Why? }\end{array}$} & $\begin{array}{l}\text { Length of } \\
\text { EH } \\
\text { (height) } \\
\text { Length of } \\
\text { EF (base) }\end{array}$ \\
\hline $\begin{array}{l}\text { Interview } \\
\text { part } 2 \\
\text { Filling } \\
\text { triangle }\end{array}$ & \multicolumn{6}{|c|}{$\begin{array}{l}\text { How does the area of } \mathrm{ABCD} \text { change as the length of } \mathrm{AD} \text { increases? } \\
\text { Imagine you created a graph relating the length of } \mathrm{AD} \text { and the area of } \mathrm{ABCD} \text {. What } \\
\text { would the graph be like? } \\
\text { Press Animate Point to sketch the graph. Was it what you expected? Use the length of } \\
\quad \mathrm{AD} \text { and the area of } \mathrm{ABCD} \text { to explain why the graph is not linear. }\end{array}$} & $\begin{array}{c}\text { Length of } \\
\text { AD } \\
\text { (height) }\end{array}$ \\
\hline
\end{tabular}

different background - a filling triangle situation. Due to time constraints, we varied only one of the aspects (height) in the new background.

\subsection{Analysis}

Whole class lessons and interviews were video recorded, transcribed, and annotated. We used two cameras during the interviews to capture both front and back views. Students' utterances, written work, and gestures served as sources of data. Employing grounded theory methodology (Corbin \& Strauss, 2008), data analysis involved multiple passes, with each pass informing subsequent passes. In the first two passes, we systematically gathered evidence of the variational and quantitative components of QVR. In each pass, we first coded separately; then, we vetted codes to come to agreement. In the third pass, we connected students' QVR to their discernment of variation in unidirectional change.

Table 2 Codes and examples: students' reasoning

Reasoning: code Example

Formulas/calculations

Static objects

Attributes in the process of changing
Daria: I just don't get it because, how do you get from this [number representing length of $A D]$ to that [number representing area of $A B C D]$ ?

Crista: It's [the triangle] half of a rectangle. Rectangles are almost all the time, linear.

Olivia: This $[D C]$ would decrease and that would make the area go smaller. 


\subsubsection{QVR: variational component}

In the first pass, we included the whole class lessons and follow-up interviews as sources of data. We used three codes: formulas/calculations, static objects, and attributes in the process of changing. Table 2 provides examples of each code. The formula/calculation code indicated when students determined or attempted to determine particular numerical amounts. For example, Daria wondered how to determine the amount of area of ABCD given an amount of length of AD (see Fig. 4). The static object code indicated when students made claims about types of shapes. For example, Crista claimed the triangle was "half of a rectangle." The attributes in the process of changing code indicated when students used utterances or gestures to describe change in progress. For example, Olivia used "go smaller" to describe change in progress. We used the theoretical construct of smooth images of change (Castillo-Garsow et al., 2013) to base our coding for change in progress. When we coded for attributes in the process of changing, we claimed those students were engaging in the variational component of QVR.

\subsubsection{QVR: quantitative component}

In the second pass, we narrowed our sources of data to those instances in which students were engaging in the variational component of QVR. We included only the follow-up interview as a source of data because students' responses during the whole class lessons did not provide a sufficiently rich source of data to make claims about students' conceptions of attributes. We used three codes for students' conceptions of attributes: physical, numerical, and measurable. We used Thompson's theory of quantitative reasoning (1994) to inform our process of coding students' conceptions of attributes. Table 3 provides examples of each code. The physical code indicated when students made claims about a shape (or an attribute of a shape). The numerical code indicated when students made claims about numerical values displayed in the DCEs (Figs. 3 and 4). The measurable code indicated when students made claims about an attribute they conceived of as being possible to measure. We separated the physical and numerical codes from the measurable code to distinguish attributes students noticed in the DCEs (e.g., pointiness, see Section 2.1) from attributes students could conceive of as possible to measure. We coded Navarro's response "physical" because he described what he noticed about the "sides" of the triangle. We coded Tomas's response "numerical," because he made claims about numerical values displayed in the DCE. The measurable code indicated when students were engaging in the quantitative component of QVR. We coded Tien's response "measurable," because she made a claim about some attribute (area) that she could conceive of as possible to measure.

Table 3 Codes and examples: conceptions of attributes that are capable of varying

Conceptions of attributes Examples

Physical

Navarro: But with this one [the triangle], these two sides are getting closer, so like, it's [the graph]going to turn the other way

Numerical

Tomas: It goes to one to ten real fast, and then once it gets to like twenty and twenty-one, it starts taking, like it goes more slowly.

Measurable

Tien: You're getting a sm-, like a smaller, well, like a smaller amount of area added. 
Using the aforementioned codes, we made three different claims about students' reasoning. If a student received the code formulas/calculations or static objects, we claimed the student was not engaging in QVR. If a student received the code in the process of changing and the code physical or numerical, we claimed the student was engaging in variational reasoning, but not QVR. Only if a student received both codes - in the process of changing and measurabledid we claim that the student was engaging in QVR. Tien's and Olivia's responses represent examples of students who received both codes (in the process of change and measurable). Therefore, we claimed that Tien and Olivia were engaging in QVR.

\subsubsection{Variation in unidirectional change}

In the third pass, we narrowed our sources of data to the interview part 2 (Table 1), when students interacted with the filling triangle DCE. We worked to draw connections between students' predictions of graph type, their engagement in QVR, and their discernment of variation in unidirectional change. We sorted students' predictions about a graph relating the length of $\mathrm{AD}$ and the area of $\mathrm{ABCD}$ (Fig. 4). We began with two categories: linear and nonlinear. In the sorting process, we added a new category: mostly linear, to capture the scope of students' predictions. Two students (Tien and Jorge) used "mostly linear" to describe a graph that to us appeared piecewise linear.

To make claims about students' discernment of variation in unidirectional change, we drew on Thompson's theory of quantitative reasoning (1994). We used comparative methods (Corbin \& Strauss, 2008) to analyze students' utterances, gestures, and written work. We analyzed for evidence that a student could conceive of the possibility of varying the intensity of the change, while holding the direction of the change invariant. For example, Tien's response (Table 3) provides evidence of her conception of variation in the intensity of change ("smaller amount of area") along with concurrent invariance in the direction of change (area being “added"). In contrast, Olivia's response (Table 2) provides evidence of a student conceiving of variation in the direction, rather than in the intensity of change (area getting "smaller"). Hence, we claimed that Tien discerned variation in unidirectional change, while Olivia discerned variation in direction of change only.

\section{Results}

We present data from the filling triangle portion of the interview. We report on the final two tasks in the interview, making predictions about a graph relating the length of $\mathrm{AD}$ and the area of $\mathrm{ABCD}$ then explaining why the dynamic graph was not linear (Table 1). In Section 4.1, we report the range of reasoning across all 14 students to address RQ1. In Sections 4.2 and 4.3, we provide interview episodes to address RQ2. In Section 4.4, we explicate a link between students' QVR and their discernment of variation in unidirectional change.

\subsection{The range of reasoning across all 14 students}

Table 4 shows the range of reasoning across all 14 students. At left, Table 4 shows the number of students making each of the three different types of predictions for the dynamic graph. At center, Table 4 shows the number of students engaging in variational reasoning (students receiving the code in the process of changing) and QVR (only students receiving both codes in 
the process of changing and measurable), before and after viewing the dynamic graph in the filling triangle DCE (Fig. 4). At right, Table 4 shows the number of students providing evidence of discernment of variation in unidirectional change.

All students who discerned variation in unidirectional change also engaged in QVR. Yet, students' engagement in QVR was not sufficient to ensure their discernment of variation in unidirectional change. Olivia - the only student who engaged in QVR after viewing the dynamic graph but did not demonstrate evidence of discerning variation in unidirectional change - provided evidence of discerning variation, rather than invariance, in the direction of change in area. Olivia's response (Table 2) occurred after she viewed the dynamic graph. She interpreted the decreasing length of DC (Fig. 4) to be causing the area to "go smaller." Rather than keeping the direction of the change invariant (increasing), Olivia compared the amount of "space" near the bottom and the top of the triangle, stating that the "curve" happens because the direction of change in area would switch "from area going bigger to like going smaller."

We found parallels between Olivia's interpretation of the dynamic graph and Jorge's "mostly linear" prediction. Jorge was the only student who engaged in QVR after, but not also before viewing the graph. When making a prediction, Jorge discerned variation in the direction of change in the "shape" of the filled region: "it's [the graph] probably going to curve a little bit, because, maybe, or be flat, because the shape is getting smaller and smaller." Furthermore, Jorge did not demonstrate evidence of discernment of variation in unidirectional change until after viewing the dynamic graph, stating: "As soon as it got to that one point, it [the area] started to get slower and slower, added on a little bit slower." The responses of Olivia and Jorge suggest that discerning variation in unidirectional change has roots in discerning variation in the direction of change. Students can build on variation in the direction of change ("going smaller") to conceive of the possibility of varying the intensity of change ("added on a little bit slower").

\subsection{Conceiving of varying an invariant attribute: affordances of QVR}

Affordances of QVR included conceiving of varying an invariant attribute. To illustrate, we share the response of Sergio, one of the four students predicting that a graph relating attributes of the filling triangle would be nonlinear. Despite being unable to vary the length of $\mathrm{AB}$ in the filling triangle DCE (see Fig. 4), some students inquired about the possibility of doing so. After viewing the dynamic filling triangle graph, without prompting from Johnson, Sergio conceived of variation in $\mathrm{AB}$, then predicted resulting changes in a graph.

Table 4 The range of reasoning across all 14 students

\begin{tabular}{|c|c|c|c|c|c|c|}
\hline \multirow[t]{2}{*}{$\begin{array}{l}\text { Students' predictions } \\
\text { of graph type }\end{array}$} & \multirow[t]{2}{*}{$\begin{array}{l}\text { Number } \\
\text { of students }\end{array}$} & \multicolumn{2}{|c|}{$\begin{array}{l}\text { Variational reasoning } \\
\text { (including those students } \\
\text { engaging in QVR) }\end{array}$} & \multicolumn{2}{|c|}{$\begin{array}{l}\text { Quantitative } \\
\text { variational } \\
\text { reasoning }(\mathrm{QVR})\end{array}$} & \multirow[t]{2}{*}{$\begin{array}{l}\text { Discernment of } \\
\text { variation in } \\
\text { unidirectional change }\end{array}$} \\
\hline & & $\begin{array}{l}\text { Before } \\
\text { viewing } \\
\text { graph }\end{array}$ & $\begin{array}{l}\text { After } \\
\text { viewing } \\
\text { graph }\end{array}$ & $\begin{array}{l}\text { Before } \\
\text { viewing } \\
\text { graph }\end{array}$ & $\begin{array}{l}\text { After } \\
\text { viewing } \\
\text { graph }\end{array}$ & \\
\hline Linear & 8 & 4 & 4 & 4 & 1 & 0 \\
\hline "Mostly linear" & 2 & 2 & 2 & 1 & 2 & 2 \\
\hline Nonlinear & 4 & 4 & 4 & 4 & 4 & 4 \\
\hline Total & 14 & 10 & 10 & 9 & 7 & 6 \\
\hline
\end{tabular}


Sergio: I'm not sure, but if you make the triangle a little bit bigger, same thing will happen but with different area.

Johnson: Why do you say that?

Sergio: Because - , um, because it's all about A and B [segment AB], Johnson: Okay

Sergio: If you change the length of $\mathrm{A}$ and $\mathrm{B}$ [segment $\mathrm{AB}$ ] then the area [of $\mathrm{ABCD}$ ] will be way different but the same structure [Traces graph shown on the computer screen] will be the same, the same structure, going, that way. [Continues to trace graph]

Johnson: Can you use the area of the triangle to explain why? I think that is an amazing observation that you are making, but, can you use that, can you tell me about the area to explain why you anticipate that?

Sergio: Because all triangles have, are like, goes in, actually, have three sides- [Motions hands to form what appears to be a vertex and sides of a triangle] And that shows it can't expand any more, like a square. So when you go up more on the triangle, then the area, [Forms what appears to be a triangle with this thumbs and index fingers] um, kind of decreases because not that much space can be inside of it.

During the filling rectangle portion of the interview and in whole class lessons, students had the opportunity to explore changes in a graph relating the length of EH and the area of EFGH for a rectangle with base (EF) of varying length (see Table 1). Sergio's response provided evidence that he could engage in a similar exploration for a filling triangle. Notably, in the filling triangle DCE, it was not possible to vary the base of the filling triangle (length of $\mathrm{AB}$ ). Yet, Sergio demonstrated that he could conceive of varying the length of $\mathrm{AB}$ and predict resulting effects on a linked graph.

Conceiving of variation in a measurable attribute of the filling triangle (length of $A B$ ), Sergio predicted resulting changes in area (i.e., "the area would be way different) as well as invariance in the shape of a related graph (i.e., "the same structure"). When introducing the term "structure," Sergio repeatedly traced the graph on the computer screen, suggesting that by "structure," he meant the shape of the graph. Because he had expressed uncertainty (i.e., "I'm not sure, but..."), Johnson encouraged Sergio to elaborate, suggesting that Sergio use area to explain his conjecture. By appealing to changes in an attribute - area - that he conceived of as measurable, Sergio demonstrated engagement in QVR across multiple attributes - the length of $\mathrm{AB}$ and the area of $\mathrm{ABCD}$.

Because Sergio treated the graph as a manipulable shape, he provided evidence of engaging in a form of static shape thinking (Moore \& Thompson, 2015). Importantly, Sergio's focus on the resulting invariance in the shape of the graph given changes in the length of $A B$ illustrates how quantitative reasoning can play a role in static shape thinking. Specifically, Sergio discerned that changing the length of $\mathrm{AB}$ would change the resulting area of $\mathrm{ABCD}$, and hence result in changes in the shape of a related graph.

\subsection{Discerning variation in unidirectional change: representing different attributes}

Students who discerned variation in unidirectional change conceived of different attributes as being possible to measure. To illustrate, we share the response of Tomas, another of the four students predicting that a graph relating attributes of the filling triangle would be nonlinear. When Johnson asked students to sketch a graph relating the length of AD and the area of 
$\mathrm{ABCD}$, Tomas created the graph shown in Fig. 5. In the transcript that follows, Johnson asked Tomas to talk more about his graph.

Johnson: Can you tell me what slower means? What going slower means in terms of area and side length?

Tomas: Um, cause at the bottom, there is a lot of like open space, [Moves pencil over shaded region on triangle diagram] and it's wide, going at a constant rate. [Runs pencil along leftmost portion of his graph] But as the side length of $\mathrm{AD}$ [Runs finger along segment $\mathrm{AD}$ ] and $\mathrm{DC}$ is nearing the top, [Runs finger along segment $\mathrm{DC}$ ] the area, like since it has less room, it increases by much less than the bottom. It goes a little bit slow. [Runs finger along his graph, beginning near the origin, slowing down when reaching the curved portion] Here it goes slower. The area goes slower, [Runs finger along the curved part of his graph] And then it goes, [Runs finger along segment AD, beginning at the base and moving up] and then it goes back down. [Lifts finger from the tip of the triangle back to the base of the triangle, then runs finger along segment AD, going up] It goes at a constant rate again. [Runs pencil along rightmost portion of his graph] Johnson: Ah, so this constant rate up here is when you come back down and graph the down part again?

Tomas: Yeah.

We had intended for students to sketch a graph representing one complete fill of the triangle. Instead, Tomas sketched a graph representing a filling triangle that began partially filled, then filled completely, and then refilled to the original partial amount. By making distinctions about how the area "goes" (e.g., "slower," "constant rate"), Tomas provided evidence that he could

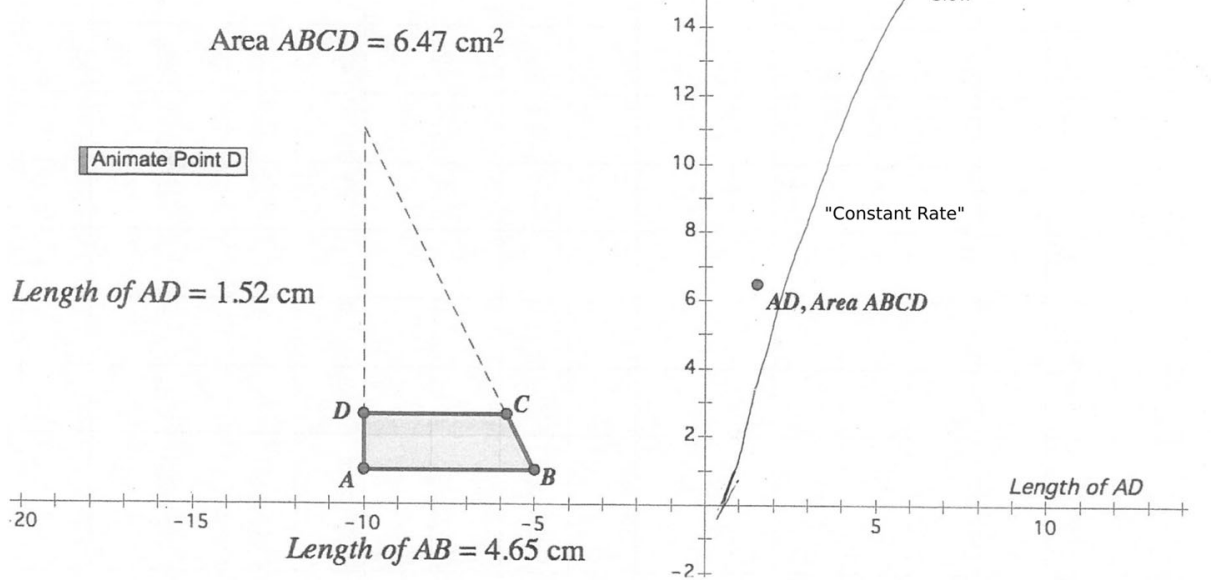

Fig. 5 Tomas's graph 
conceive of variation in the intensity of the speed at which he perceived the area to be changing. Moving back and forth between his graph and the diagram of ABCD (Fig. 5), Tomas provided evidence that his graph represented variation in an attribute he could conceive of as possible to measure. For example, after Tomas stated: "it [area] increases by much less than the bottom," he ran his finger along a portion of his graph to show when area was changing in the way he described. Therefore, we claim that Tomas discerned variation in unidirectional change.

Instead of using a graph to represent a relationship between two quantities capable of varying, we interpret that Tomas used a graph to represent variation in a single quantity. Therefore, we claim that Tomas engaged in variational, rather than covariational reasoning. Because Tomas could conceive of variation in an attribute, we interpret him to be reasoning at a level that Thompson and Carlson (2017) term gross variation. Furthermore, Tomas described the area as being in the process of changing (e.g., "it goes a little bit slow."). Hence, we claim that Tomas demonstrated smooth images of change. Consequently, Tomas's response provides evidence that a student engaging in variational reasoning at the level of gross variation can also demonstrate smooth images of change.

Tomas demonstrated that he could interpret a graph as a trace representing the speed at which area was changing as an animation elapsed in real time. For example, when Tomas said: "Here it goes slower. The area goes slower," he ran his finger along a portion of his graph. In our view, Tomas's reasoning is consistent with aspects of the construct of emergent shape thinking, put forward by Moore and Thompson (2015). Going further, we interpret Tomas to be engaging in a form of emergent shape thinking that involves variational, rather than covariational reasoning.

\subsection{A link between students' QVR and their discernment of variation in unidirectional change}

All students who discerned variation in unidirectional change also engaged in QVR. Yet, students' use of QVR was not sufficient to engender their discernment of variation in unidirectional change (e.g., Olivia). We cannot rule out the possibility for students to discern variation in unidirectional change without engaging in QVR. For example, students may discern variation in unidirectional change in attributes they notice in DCEs (e.g., a triangle becoming "more" pointy). Despite this possibility, all students who discerned variation in unidirectional change also provided evidence that they were attending to attributes they could conceive of as possible to measure. Interestingly, the attributes were not necessarily attributes we expected (e.g., Tomas) or attributes directly represented in a graph (e.g., Sergio). Furthermore, all students discerning variation in unidirectional change also demonstrated smooth images of change.

\section{Discussion}

\subsection{The utility of smooth images of change}

To date, researchers conducting empirical investigations of secondary students' smooth images of change have investigated students' covariational reasoning (e.g., CastilloGarsow et al., 2013; Johnson, 2012). Our results provide evidence to support the utility 
of students' smooth images of change for their variational reasoning as well. Furthermore, we build on previous research (Ellis et al., 2015; Lobato et al., 2012) by providing evidence of early secondary students' use of smooth images of change to discern variation in unidirectional change. Our results demonstrate how students can be using smooth images of change while engaging in variational reasoning at the gross variation level (Thompson \& Carlson, 2017).

\subsection{Extending constructs of static and emergent shape thinking}

Our analysis of Tomas and Sergio's responses suggest two extensions to Moore and Thompson's (2015) constructs of static and emergent shape thinking. First, static shape thinking can have quantitative aspects. Furthermore, when engaging in forms of static shape thinking, students can reason about quantities not directly represented in graphs they interpret or create.

Second, emergent shape thinking can take a variational form. To be clear, we do not propose that "variational" emergent shape thinking is an end goal for students. Rather, we intend to highlight the promise of "variational" emergent shape thinking in fostering students' development of covariational reasoning.

\subsection{Task design principles to foster students' discernment of variation in unidirectional change}

As did Johnson (2012), we distinguish between tasks involving variation within quantities (e.g., filling triangle tasks, Table 1) and tasks involving variation between quantities (e.g., filling rectangle tasks, Table 1). By moving from the filling rectangle to the filling triangle situation, we intended to provide students opportunities to discern variation in unidirectional change within a particular instance of a filling triangle rather than only between instances of filling rectangles. Through our task design and sequencing, the filling triangle situation provided a specialized kind of contrast (Marton, 2015) that extended beyond a difference in shape (triangle vs. rectangle) to a different kind of variation. Furthermore, our results provide evidence to support Stroup's (2002) assertion that a linear situation may be "too simple" to foster students' attention to complexities underlying key concepts such as rate. In particular, we argue that linear situations alone are insufficient to use to provide students opportunities to discern variation in unidirectional change, because the variation in unidirectional change only occurs between rather than within instances.

\section{Summary}

In summary, the main findings of this study include two factors related to students' discernment of variation in unidirectional change. The first factor is students' conceptions of attributes as "things" that are capable of varying and possible to measure. The second factor is students' use of smooth images of change when engaging in variational reasoning. In this study, the only students who discerned variation in unidirectional change were those students who engaged in QVR and demonstrated smooth images of change.

To situate our findings, we acknowledge that it would be appropriate for students to use covariational reasoning when interacting with the filling polygon DCEs and related tasks that we 
included. By investigating the variational reasoning that these students used, rather than focusing on students' lack of covariational reasoning, we were able to learn more about the richness of early secondary students' mathematical reasoning. In our view, providing students opportunities to engage in QVR is useful for promoting their engagement in covariational reasoning.

\section{Implications for design of tasks and DCEs}

This study has two implications for task design to foster students' reasoning about the mathematics of change and variation. First, opportunities to interact with DCEs linking animations and dynamic graphs are insufficient to foster students' discernment of variation in unidirectional change. When designing DCEs and related tasks, incorporate attributes that students can conceive of as capable of varying and possible to measure. Second, students' conceptions of change can impact their variational reasoning when interacting with DCEs. To foster students' discernment of variation in unidirectional change, incorporate tasks that provide opportunities for students to use smooth images of change.

Acknowledgements We thank Kevin Moore, Patrick Thompson, Eva Thanheiser, Amber Gardner, Daniel Grieser, and reviewers for their comments on previous versions of this manuscript.

Funding information This research was supported in part by a Faculty Development Grant from the University of Colorado Denver.

Compliance with ethical standards Opinions, findings, and conclusions are those of the authors. Participants and their parent or guardian gave written informed consent to participate in this research.

Open Access This article is distributed under the terms of the Creative Commons Attribution 4.0 International License (http://creativecommons.org/licenses/by/4.0/), which permits unrestricted use, distribution, and reproduction in any medium, provided you give appropriate credit to the original author(s) and the source, provide a link to the Creative Commons license, and indicate if changes were made.

\section{References}

Ayalon, M., Watson, A., \& Lerman, S. (2015). Functions represented as linear sequential data: Relationships between presentation and student responses. Educational Studies in Mathematics, 90(3), 321-339. https://oi.org/10.1007/s10649-015-9628-9

Bell, A., \& Janvier, C. (1981). The interpretation of graphs representing situations. For the Learning of Mathematics, 2(1), 34-42.

Carlson, M. P., Jacobs, S., Coe, E., Larsen, S., \& Hsu, E. (2002). Applying covariational reasoning while modeling dynamic events: A framework and a study. Journal for Research in Mathematics Education, 33(5), $352-378$.

Castillo-Garsow, C., Johnson, H. L., \& Moore, K. C. (2013). Chunky and smooth images of change. For the Learning of Mathematics, 33(3), 31-37.

Clement, J. (1989). The concept of variation and misconceptions in cartesian graphing. Focus on Learning Problems in Mathematics, 11(1-2), 77-87.

Clement, J. (2000). Analysis of clinical interviews: Foundations and model viability. In A. E. Kelly \& R. A. Lesh (Eds.), Handook of research design in mathematics and science education (pp. 547-589). Mahwah, NJ: Lawrence Erlbaum Associates.

Cobb, P., Confrey, J., diSessa, A., Lehrer, R., \& Schauble, L. (2003). Design experiments in educational research. Educational Researcher, 32(1), 9-13. 
Confrey, J., \& Smith, E. (1995). Splitting, covariation, and their role in the development of exponential functions. Journal for Research in Mathematics Education, 26(1), 66-86.

Corbin, J., \& Strauss, A. (2008). Basics of qualitative research: Techniques and procedures for developing grounded theory (3rd ed.). London: Sage Publications.

Ellis, A. B., Özgür, Z., Kulow, T., Williams, C., \& Amidon, J. (2015). Quantifying exponential growth: Three conceptual shifts in coordinating multiplicative and additive growth. Journal of Mathematical Behavior, 39, $131-155$.

Herbert, S., \& Pierce, R. (2012). Revealing educationally critical aspects of rate. Educational Studies in Mathematics, 81(1), 85-101.

Hitt, F., \& González-Martín, A. S. (2015). Covariation between variables in a modelling process: The ACODESA (collaborative learning, scientific debate and self-reflection) method. Educational Studies in Mathematics, 88(2), 201-219. https://doi.org/10.1007/s10649-014-9578-7

Jackiw, N. (2009). The geometer's sketchpad (version 5.0) [computer software]. Emeryville, CA: Key Curriculum Technologies.

Johnson, H. L. (2012). Reasoning about variation in the intensity of change in covarying quantities involved in rate of change. Journal of Mathematical Behavior, 31(3), 313-330.

Johnson, H. L. (2015a). Secondary students' quantification of ratio and rate: A framework for reasoning about change in covarying quantities. Mathematical Thinking and Learning, 17(1), 64-90.

Johnson, H. L. (2015b). Together yet separate: Students' associating amounts of change in quantities involved in rate of change. Educational Studies in Mathematics, 89(1), 89-110. https://doi.org/10.1007/s10649-0149590-y

Johnson, H. L., McClintock, E., Hornbein, P., Gardner, A., \& Grieser, D. (2017). When a critical aspect is a conception: Using multiple theories to design dynamic computer environments and tasks to foster students' discernment of covariation. 10th Congress of European Research in Mathematics Education, Dublin, Ireland. Retrieved November 9, 2017 from https://keynote.conference-services.net/resources/444/5118 /pdf/CERME10_0124.pdf

Kaput, J. J. (1994). Democratizing access to calculus: New routes to old roots. In A. H. Schoenfeld (Ed.), Mathematical thinking and problem solving (pp. 77-156). Hillsdale, New Jersey: Lawrence Erlbaum Associates.

Leinhardt, G., Zaslavsky, O., \& Stein, M. K. (1990). Functions, graphs, and graphing: Tasks, learning and teaching. Review of Educational Research, 60(1), 1-64.

Lobato, J., Hohensee, C., Rhodehamel, B., \& Diamond, J. (2012). Using student reasoning to inform the development of conceptual learning goals: The case of quadratic functions. Mathematical Thinking and Learning, 14(2), 85-119.

Marton, F. (2015). Necessary conditions of learning. New York: Routledge.

Moore, K. C., \& Thompson, P. W. (2015). Shape thinking and students' graphing activity. In T. FukawaConnelly (Ed.), Proceedings of the 18th Meeting of the MAA Special Interest Group on Research in Undergraduate Mathematics Education (pp. 782-789). Pittsburgh, PA: RUME.

Saldanha, L., \& Thompson, P. W. (1998). Re-thinking covariation from a quantitative perspective: Simultaneous continuous variation. In S. B. Berenson, K. R. Dawkins, M. Blanton, W. N. Coloumbe, J. Kolb, K. Norwood, \& L. Stiff (Eds.), Proceedings of the 20th annual meeting of the Psychology of Mathematics Education North American Chapter (Vol. 1, pp. 298-303). Raleigh, NC: North Carolina State University.

Shell Centre for Mathematical Education (University of Nottingham). (1985). The language of functions and graphs: An examination module for secondary schools. Nottingham: JMB/Shell Centre for Mathematical Education.

Slavit, D. (1997). An alternate route to the reification of function. Educational Studies in Mathematics, 33(3), $259-281$.

Stroup, W. (2002). Understanding qualitative calculus: A structural synthesis of learning research. International Journal of Computers for Mathematical Learning, 7, 167-215.

Thompson, P. W. (1994). The development of the concept of speed and its relationship to concepts of rate. In G. Harel \& J. Confrey (Eds.), The development of multiplicative reasoning in the learning of mathematics (pp. 181-234). Albany, NY: State University of New York Press.

Thompson, P. W. (1996). Imagery and the development of mathematical reasoning. In L. P. Steffe, P. Nesher, P. Cobb, G. A. Goldin, \& B. Greer (Eds.), Theories of mathematical learning (pp. 267-284). Mahwah, NJ: Lawrence Erlbaum Associates.

Thompson, P. W., Byerley, C., \& Hatfield, N. (2013). A conceptual approach to calculus made possible by technology. Computers in Schools, 30, 124-147.

Thompson, P. W., \& Carlson, M. P. (2017). Variation, covariation and functions: Foundational ways of mathematical thinking. In J. Cai (Ed.), Compendium for research in mathematics education (pp. 421456). Reston, VA: National Council of Teachers of Mathematics. 